\title{
Cayley graphs on the symmetric group generated by initial reversals have unit spectral gap
}

\author{
Filippo Cesi \\ Dipartimento di Fisica \\ Università di Roma "La Sapienza", Italy \\ and SMC, INFM-CNR. \\ filippo.cesi@roma1.infn.it \\ Submitted: Apr 11, 2009; Accepted: Oct 1, 2009; Published: Oct 12, 2009 \\ Mathematics Subject Classification: 05C25, 05C50
}

\begin{abstract}
In a recent paper Gunnells, Scott and Walden have determined the complete spectrum of the Schreier graph on the symmetric group corresponding to the Young subgroup $S_{n-2} \times S_{2}$ and generated by initial reversals. In particular they find that the first nonzero eigenvalue, or spectral gap, of the Laplacian is always 1, and report that "empirical evidence" suggests that this also holds for the corresponding Cayley graph. We provide a simple proof of this last assertion, based on the decomposition of the Laplacian of Cayley graphs, into a direct sum of irreducible representation matrices of the symmetric group.
\end{abstract}

\section{Introduction}

If $G$ is a finite group, $H$ is a subgroup of $G$ and $Z$ is a generating set of $G$, we can construct the Schreier graph $\mathcal{G}=X(G, H, Z)$ whose vertices are the left-cosets $G / H$, and whose edges are the pairs $(g H, z g H)$ with $g H \in G / H$ and $z \in Z$. We assume that the generating set $Z$ is symmetric, i.e. $z \in Z$ if and only if $z^{-1} \in Z$. In this case the graph $X(G, H, Z)$ is undirected. If $H=\{1\}$ we denote with $X(G, Z)=X(G,\{1\}, Z)$ the Cayley graph of $G$ associated to the generating set $Z$. If $A_{\mathcal{G}}$ is the adjacency matrix of $\mathcal{G}$ and $\Delta_{\mathcal{G}}$ the corresponding Laplacian, since $\mathcal{G}$ is $|Z|$-regular (counting loops), we have

$$
\Delta_{\mathcal{G}}=|Z| I-A_{\mathcal{G}},
$$

where $|Z|$ stands for the cardinality of the set $Z$. The Laplacian is symmetric and positivesemidefinite, hence its eigenvalues are real and nonnegative and can be ordered as

$$
0=\lambda_{1}\left(\Delta_{\mathcal{G}}\right) \leqslant \lambda_{2}\left(\Delta_{\mathcal{G}}\right) \leqslant \cdots \leqslant \lambda_{n}\left(\Delta_{\mathcal{G}}\right)
$$


Since $Z$ generates $G$, the graph $\mathcal{G}$ is connected, which implies that 0 is a simple eigenvalue with constant eigenvector, while $\lambda_{2}\left(\Delta_{\mathcal{G}}\right)$ is strictly positive. The second eigenvalue of the Laplacian is also called the spectral gap of the graph $\mathcal{G}$ and we denote it with gap $\mathcal{G}$. For regular graphs, it coincides with the difference between the two largest eigenvalues of the adjacency matrix.

In [6] the authors consider the Schreier graph $X\left(S_{n}, S_{(n-2,2)}, Z_{n}\right)$ where $S_{n}$ is the symmetric group, $S_{(n-2,2)}$ is the Young subgroup corresponding to the partition $(n-2,2)$, isomorphic to $S_{n-2} \times S_{2}$, and $Z_{n}:=\left\{r_{1}, \ldots, r_{n}\right\}$, where $r_{k}$ is the permutation which reverses the order of the first $k$ positive integers

$$
r_{k}:\{1,2, \ldots, n\} \longrightarrow\{k, k-1, \ldots, 2,1, k+1, \ldots, n\}
$$

In [6] the spectrum of the Laplacian was determined, and it turns out that

$$
\operatorname{spec} \Delta_{X\left(S_{n}, S_{(n-2,2)}, Z_{n}\right)} \subset\{0,1, \ldots, n\}
$$

with equality if $n \geqslant 8$. It was also proved that gap $X\left(S_{n}, S_{(n-2,2)}, Z_{n}\right)=1$ for all $n \geqslant 3$. On the other side it was shown in [10] that, if $Z_{n}$ is a set of reversals with $\left|Z_{n}\right|=o(n)$, then gap $X\left(S_{n}, S_{(n-2,2)}, Z_{n}\right) \rightarrow 0$ as $n \rightarrow \infty$. Hence, results in [6] show that condition $\left|Z_{n}\right|=o(n)$ is, in a sense, optimal.

It is easy to see that if $K$ is a subgroup of $H$, then the spectrum of $X(G, H, Z)$ is a subset of the spectrum of $X(G, K, Z)$. In particular the spectrum of $X(G, H, Z)$ is a subset of the spectrum of the Cayley graph $X(G, Z)$, thus we have gap $X(G, Z) \leqslant$ gap $X(G, H, Z)$. By consequence we get, for what concerns the symmetric group generated by initial reversals, ${ }^{1}$

$$
\operatorname{gap} X\left(S_{n}, Z_{n}\right) \leqslant \operatorname{gap} X\left(S_{n}, S_{(n-2,2)}, Z_{n}\right)=1 \quad n \geqslant 3 .
$$

Our main result confirms what in [6] was indicated as "empirical evidence".

Theorem 1.1. Let $Z_{n}:=\left\{r_{1}, \ldots, r_{n}\right\}$ be the set of all initial reversals defined in (1.1). Then, for any $n \geqslant 3$, we have gap $X\left(S_{n}, Z_{n}\right)=1$.

Our approach is based on the connection between the Laplacian of a Cayley graph for a finite group $G$ and the irreducible representations of $G$. A similar approach has allowed a detailed study of the spectrum of Cayley graphs on $S_{n}$ generated by a set of transpositions $Z$, when $Z$, interpreted as as the edge set of a graph with $n$ vertices, yields a complete graph [4], or a complete multipartite graph [3].

\section{Cayley graphs and irreducible representations}

In this section we introduce our notation and briefly recall a few basic facts about the eigenvalues of (weighted) Cayley graphs and the irreducible representations of a finite

\footnotetext{
${ }^{1}$ when $n=2$ a trivial computation yields $\operatorname{gap}\left(S_{2}, Z_{2}\right)=2$.
} 
group. Details can be found for instance in [8]. Let $\mathbf{Y}$ be a representation of a finite group $G$. $\mathbf{Y}$ extends to a representation of the complex group algebra $\mathbb{C} G$ by letting $\mathbf{Y}(w):=\sum_{g \in G} w_{g} \mathbf{Y}(g)$, where $w=\sum_{g \in G} w_{g} g$ is an element in $\mathbb{C} G$. $\operatorname{Irr}(G)$ stands for the set of all equivalence classes of irreducible representations of $G$. If $[\alpha] \in \operatorname{Irr}(G)$ we denote with $\mathbf{T}^{\alpha}$ a specific choice in the class $[\alpha]$. By Maschke's complete reducibility theorem, any representation $\mathbf{Y}$ is equivalent to a direct sum

$$
\mathbf{Y} \cong \bigoplus_{[\alpha] \in \operatorname{Irr}(G)} y_{\alpha} \mathbf{T}^{\alpha}
$$

where $y_{\alpha}$ are suitable nonnegative integers. By consequence the spectrum of $\mathbf{Y}(w)$ is just the union of the spectra of those $\mathbf{T}^{\alpha}(w)$ for which $y_{\alpha}$ is nonzero. ${ }^{2}$ We define the set of all symmetric elements in $\mathbb{C} G$ and the set of all symmetric nonnegative elements as

$$
\begin{aligned}
\operatorname{Sym} \mathbb{C} G & :=\left\{w \in \mathbb{C} G: w_{g}=\bar{w}_{g^{-1}} \text { for all } g \in G\right\} \\
(\operatorname{Sym} \mathbb{C} G)_{+} & :=\left\{w \in \operatorname{Sym} \mathbb{C} G: w_{g} \in \mathbb{R}, w_{g} \geqslant 0 \text { for all } g \in G\right\} .
\end{aligned}
$$

If $\mathbf{Y}$ is a unitary representation and $w$ is symmetric, then $\mathbf{Y}(w)$ is a Hermitian matrix. Since every representation of a finite group is equivalent to a unitary representation, the eigenvalues of $\mathbf{Y}(w)$ are real for any representation $\mathbf{Y}$ and for any $w \in \operatorname{Sym} \mathbb{C} G$. We denote with $\lambda_{\max }(\mathbf{Y}(w))$ the largest eigenvalue of $\mathbf{Y}(w)$. A trivial upper bound on this quantity is found by assuming $\mathbf{Y}$ unitary

$$
\lambda_{\max }(\mathbf{Y}(w)) \leqslant\|\mathbf{Y}(w)\| \leqslant \sum_{g \in G}\left|w_{g}\right|\|\mathbf{Y}(g)\|=\sum_{g \in G}\left|w_{g}\right|=:|w|,
$$

where $\|A\|$ stands for the $\ell^{2}$ operator norm of the matrix $A$ and $|w|$ for the $\ell^{1}$ norm on $\mathbb{C} G$.

If $Z$ is a generating set for $G$ we can define an element of the group algebra $\mathbb{C} G$, which we denote by $\widehat{Z}$, given by

$$
\widehat{Z}:=\sum_{z \in Z} z .
$$

In the following we will consider symmetric generating sets $Z$, that is such that $z \in Z$ iff $z^{-1} \in Z$. In this case $\widehat{Z}$ is an element of $(\operatorname{Sym} \mathbb{C} G)_{+}$. Conversely if $w=\sum_{g \in G} w_{g} g$ is a symmetric nonnegative element in $\mathbb{C} G$, we can define the (undirected) weighted Cayley graphs $X(G, w)$, where $w_{g}$ represents the weight associated to each edge $(h, g h), h \in G$. The adjacency matrix and the Laplacian of $X(G, w)$ are closely related to the left regular representation of $G$. If we denote with $\mathbf{R}$ such a representation, it follows from the definitions that

$$
A_{X(G, w)}=|w| I-\Delta_{X(G, w)}=\mathbf{R}(w) \quad w \in(\operatorname{Sym} \mathbb{C} G)_{+} .
$$

Consider now the case in which $G$ is the permutation group $S_{n}$. There is a one-to-one correspondence between $\operatorname{Irr}\left(S_{n}\right)$ and the set of all partitions of $n$. A partition of $n$ is

\footnotetext{
${ }^{2}$ if one is interested in multiplicities of the eigenvalues, spectra must be treated as multisets.
} 
a nonincreasing sequence of positive integers $\alpha=\left(\alpha_{1}, \alpha_{2}, \ldots, \alpha_{r}\right)$ such that $\sum_{i=1}^{r} \alpha_{i}=$ $n$. We write $\alpha \vdash n$ if $\alpha$ is a partition of $n$. We denote with $[\alpha]$ the class of irreducible representations of $S_{n}$ corresponding to the partition $\alpha$. For simplicity we write $\left[\alpha_{1}, \ldots, \alpha_{r}\right]$ instead of $\left[\left(\alpha_{1}, \ldots, \alpha_{r}\right)\right]$.

Since all irreducible representations appear in the decomposition of the left regular representation, it follows from (2.3) that if we let

$$
\psi([\alpha], w):=|w|-\lambda_{\max }\left(\mathbf{T}^{\alpha}(w)\right) \quad \alpha \vdash n,
$$

then the spectral gap of $X\left(S_{n}, w\right)$ is given by

$$
\operatorname{gap} X\left(S_{n}, w\right)=\min _{\alpha \vdash n: \alpha \neq(n)} \psi([\alpha], w),
$$

where $\alpha=(n)$ is the one-dimensional identity representation which yields one eigenvalue equal to $|w|$ in $\mathbf{R}(w)$.

We conclude this section with a remark concerning a connection between results like Theorem 1.1 and Aldous's conjecture [1] asserting that the random walk and the interchange process have the same spectral gap on any finite graph. In order to explain this connection we introduce a property, which we call property (A) which is an attribute of certain elements of the group algebra: given $w \in\left(\operatorname{Sym} \mathbb{C} S_{n}\right)_{+}$, we say that property (A) holds for $w$ if one of the following two equivalent statements is satisfied

(A1) If $\alpha \vdash n$ and $\alpha \neq(n)$, then $\lambda_{\max }\left(\mathbf{T}^{\alpha}(w)\right) \leqslant \lambda_{\max }\left(\mathbf{T}^{(n-1,1)}(w)\right)$

(A2) $\operatorname{gap} X\left(S_{n}, w\right)=\psi([n-1,1], w)$.

The two statements are equivalent in virtue of (2.5). Aldous's conjecture, originally formulated in the framework of continuous time Markov chains, is equivalent (see [3]) to the assertion that: if $w=\sum_{\ell} t_{\ell}$ is a sum of transpositions $t_{\ell}=\left(i_{\ell} j_{\ell}\right) \in S_{n}$, then $w$ has property (A). A stronger "weighted graphs" version of this conjecture can be formulated in which $w=\sum_{\ell} w_{\ell} t_{\ell}$ is allowed to be a linear combination of transpositions with nonnegative coefficients. A weaker version of this statement, namely for bipartite graphs, was also conjectured in [5]. Several papers have appeared with proofs of Aldous's conjecture for some particular classes of graphs, and recently a beautiful general proof has been found by Caputo, Liggett and Richthammer [2] (see also this paper for references to previous work). Going back to our problem of finding the spectral gap of the Cayley graph $X\left(S_{n}, \widehat{Z}_{n}\right)$, where $Z_{n}$ is the set of initial reversals, we observe that Proposition 4.1 in [6] implies that $\psi\left([n-1,1], \widehat{Z}_{n}\right)=1$, hence Theorem 1.1 is equivalent to the assertion that $\widehat{Z}_{n}$ has property $(\mathrm{A})$.

\section{Proof of Theorem 1.1}

We start with a general lower bound on the spectral gap of a weighted Cayley graph $X\left(S_{n}, w_{n}\right)$ which makes use of the branching rule [11, Section 2.8] for the decomposition 
of the restriction of an irreducible representation $[\alpha]$ of $S_{n}$ to the subgroup $S_{n-1}$. This rule states that

$$
[\alpha] \downarrow_{S_{n-1}}^{S_{n}}=\bigoplus_{\beta \in \alpha^{-}}[\beta] \quad \alpha \vdash n
$$

where, if $\alpha=\left(\alpha_{1}, \ldots, \alpha_{r}\right), \alpha^{-}$is defined as the collection of all sequences of the form

$$
\left(\alpha_{1}, \ldots, \alpha_{i-1}, \alpha_{i}-1, \alpha_{i+1}, \ldots, \alpha_{r}\right)
$$

which are partitions of $n-1$. For example

$$
[6,5,5,3,1] \downarrow_{S_{19}}^{S_{20}}=[5,5,5,3,1] \oplus[6,5,4,3,1] \oplus[6,5,5,2,1] \oplus[6,5,5,3] .
$$

We have then the following lower bound on the spectral gap of $X\left(S_{n}, w_{n}\right)$.

Lemma 3.1. Let $z_{k} \in\left(\operatorname{Sym} \mathbb{C} S_{k}\right)_{+}$for $k=1,2, \ldots$, and let $w_{n}:=\sum_{k=1}^{n} z_{k}$. Then

$$
\operatorname{gap} X\left(S_{n}, w_{n}\right) \geqslant \min _{k=2, \ldots, n} \psi\left([k-1,1], w_{k}\right) \text {. }
$$

Remark 3.2. Given $w_{n}=\sum_{\pi \in S_{n}} w_{n, \pi} \pi \in\left(\operatorname{Sym} \mathbb{C} S_{n}\right)_{+}$, it is always possible to write $w_{n}$ as a sum of $z_{k}$ such that Lemma 3.1 applies. For instance one can define

$$
z_{k}=\sum_{\pi \in S_{k} \backslash S_{k-1}} w_{n, \pi} \pi
$$

even though it is not clear that this choice gives the optimal lower bound in (3.1).

Remark 3.3. Consider the case in which $w_{n}=\sum_{\ell} w_{n, \ell} t_{\ell}$ is a linear combination of transpositions $t_{\ell} \in S_{n}$ with $w_{n, \ell} \geqslant 0$, and define the graph $\mathcal{G}_{w_{n}}$ with vertex set $\{1, \ldots, n\}$ and edge set given by $\operatorname{supp} w_{n}=\left\{t_{\ell}: w_{n, \ell}>0\right\}$, in which each transposition $t_{\ell}=(i j)$ is identified with the corresponding edge $\{i, j\}$. In the case of transpositions Lemma 3.1 is equivalent to Lemma 2 in [9] and it was more or less implicit already in [7], where it was used to prove Aldous's conjecture for trees, meaning for all $w$ such that $\mathcal{G}_{w}$ is a tree. Using this approach, Aldous's conjecture has been proved independently in [9] and [12] for hypercubes asymptotically, i.e. in the limit when the side length of the cube tends to infinity. While the proof of Lemma 2 in [9] (or the equivalent statement in [7]) is not hard, it is nevertheless interesting to realize that our general formulation of this result is a direct consequence of very general algebraic identities (equality (2.5) and the branching rule).

Proof of Lemma 3.1. If $A$ and $B$ are two Hermitian $n \times n$ matrices we have

$$
\lambda_{\max }(A+B)=\max _{x \in \mathbb{C}^{n}:\|x\|=1}\langle(A+B) x, x\rangle \leqslant \lambda_{\max }(A)+\lambda_{\max }(B),
$$

where $\|x\|$ is the Euclidean norm. Using this fact and the trivial bound (2.2), we find

$$
\begin{aligned}
\lambda_{\max }\left(\mathbf{T}^{\alpha}\left(w_{n}\right)\right) & \leqslant \lambda_{\max }\left(\mathbf{T}^{\alpha}\left(w_{n-1}\right)\right)+\lambda_{\max }\left(\mathbf{T}^{\alpha}\left(z_{n}\right)\right) \\
& \leqslant \lambda_{\max }\left(\mathbf{T}^{\alpha}\left(w_{n-1}\right)\right)+\left|z_{n}\right| .
\end{aligned}
$$


Since $w_{n-1} \in\left(\operatorname{Sym} \mathbb{C} S_{n-1}\right)_{+}$, we can write $\mathbf{T}^{\alpha}\left(w_{n-1}\right) \cong \bigoplus_{\beta \in \alpha^{-}} \mathbf{T}^{\beta}\left(w_{n-1}\right)$, thus

$$
\lambda_{\max }\left(\mathbf{T}^{\alpha}\left(w_{n-1}\right)\right)=\max _{\beta \in \alpha^{-}} \lambda_{\max }\left(\mathbf{T}^{\beta}\left(w_{n-1}\right)\right) .
$$

It follows from the branching rule that if $\alpha \neq(n)$ and $\alpha \neq(n-1,1)$, then the trivial partition $(n-1)$ is not contained in $\alpha^{-}$. By consequence

$$
\max _{\substack{\alpha \vdash n \\ \alpha \neq(n), \alpha \neq(n-1,1)}} \lambda_{\max }\left(\mathbf{T}^{\alpha}\left(w_{n}\right)\right) \leqslant \max _{\substack{\beta \vdash n-1: \\ \beta \neq(n-1)}} \lambda_{\max }\left(\mathbf{T}^{\beta}\left(w_{n-1}\right)\right)+\left|z_{n}\right| .
$$

Since $z_{k}$ and $w_{n}$ have nonnegative components, we get

$$
\left|w_{n}\right|:=\sum_{\pi \in S_{n}} w_{n, \pi}=\sum_{\pi \in S_{n}}\left(w_{n-1, \pi}+z_{n, \pi}\right)=\left|w_{n-1}\right|+\left|z_{n}\right| .
$$

From (2.5), (3.2) and (3.3) we obtain

$$
\begin{aligned}
& \operatorname{gap} X\left(S_{n}, w_{n}\right)=\min _{\alpha \vdash n: \alpha \neq(n)}\left[\left|w_{n}\right|-\lambda_{\max }\left(\mathbf{T}^{\alpha}\left(w_{n}\right)\right)\right] \\
& \geqslant \min \left\{\min _{\substack{\beta \vdash n-1: \\
\beta \neq(n-1)}}\left[\left|w_{n-1}\right|-\lambda_{\max }\left(\mathbf{T}^{\beta}\left(w_{n-1}\right)\right)\right], \psi\left([n-1,1], w_{n}\right)\right\} .
\end{aligned}
$$

Hence we get the recursive inequality

$$
\operatorname{gap} X\left(S_{n}, w_{n}\right) \geqslant \min \left\{\operatorname{gap} X\left(S_{n-1}, w_{n-1}\right), \psi\left([n-1,1], w_{n}\right)\right\} \text {. }
$$

When $n=2$ we have $S_{2}=\{1,(12)\}$ and $w_{2}=w_{2,1} \cdot 1+w_{2,(12)} \cdot(12)$. A trivial computation yields

$$
\operatorname{gap} X\left(S_{2}, w_{2}\right)=\psi\left([1,1], w_{2}\right)=2 w_{2,(12)}
$$

which, together with (3.5), implies the Lemma.

Proof of Theorem 1.1. Let $r_{k}$ be the permutation which reverse the order of the first $k$ positive integers

$$
r_{k}:\{1,2, \ldots, n\} \longrightarrow\{k, k-1, \ldots, 2,1, k+1, \ldots, n\},
$$

and let $w_{n}:=\sum_{k=1}^{n} r_{k}$. Let also $\mathbf{D}_{n}$ be the $n$-dimensional defining representation of $S_{n}$, which can be written as

$$
\left[\mathbf{D}_{n}\right]=[n] \oplus[n-1,1] .
$$

The eigenvalues and eigenvectors of the matrix $\mathbf{D}_{n}\left(w_{n}\right)$ are listed in [6, Proposition 4.1]. The two largest eigenvalues are $n$ and $n-1$, both simple. Since the eigenvalue $n$ clearly corresponds to the identity representation $[n]$ contained in $\mathbf{D}_{n}$, we have, for each $n \geqslant 3$,

$$
\lambda_{\max }\left(\mathbf{T}^{(n-1,1)}\left(w_{n}\right)\right)=n-1 .
$$

By consequence,

$$
\psi\left([n-1,1], w_{n}\right)=1 \quad n \geqslant 3 .
$$

From Lemma 3.1, (1.2) and from (3.6) which in this case says $\psi\left([1,1], w_{2}\right)=2$, it follows that $\operatorname{gap}\left(S_{n}, w_{n}\right)=1$. 


\section{References}

[1] David Aldous, www.stat.berkeley.edu/〜aldous/research/op/sgap.html.

[2] Pietro Caputo, Thomas M. Liggett, and Thomas Richthammer, A recursive proof of Aldous' spectral gap conjecture, arXiv:0906.1238v3 (2009).

[3] Filippo Cesi, On the eigenvalues of Cayley graphs on the symmetric group generated by a complete multipartite set of transpositions, arxiv:0902.0727v1 (2009).

[4] Persi Diaconis and Mehrdad Shahshahani, Generating a random permutation with random transpositions, Z. Wahrsch. Verw. Gebiete 57 (1981), no. 2, 159-179.

[5] Joel Friedman, On Cayley graphs on the symmetric group generated by transpositions, Combinatorica 20 (2000), no. 4, 505-519.

[6] Paul E. Gunnells, Richard A. Scott, and Byron L. Walden, On certain integral Schreier graphs of the symmetric group, Electron. J. Combin. 14 (2007), no. 1, Research Paper 43, 26 pp. (electronic).

[7] Shirin Handjani and Douglas Jungreis, Rate of convergence for shuffling cards by transpositions, J. Theoret. Probab. 9 (1996), no. 4, 983-993.

[8] Alexander Lubotzky, Cayley graphs: eigenvalues, expanders and random walks, Surveys in combinatorics, 1995 (Stirling), London Math. Soc. Lecture Note Ser., vol. 218, Cambridge Univ. Press, Cambridge, 1995, pp. 155-189.

[9] Ben Morris, Spectral gap for the interchange process in a box, Electron. Commun. Probab. 13 (2008), 311-318.

[10] D. Nash, Cayley graphs of symmetric groups generated by reversals, Pi Mu Epsilon Journal 12 (2005), 143-147.

[11] Bruce E. Sagan, The symmetric group: Representations, combinatorial algorithms, and symmetric functions, second ed., Graduate Texts in Mathematics, vol. 203, Springer-Verlag, New York, 2001.

[12] Shannon Starr and Matt Conomos, Asymptotics of the spectral gap for the interchange process on large hypercubes, arxiv:0802.1368v2 (2008). 